\title{
Direct MR arthrography of the shoulder: current practice in the UK
}

\author{
J. Kho ${ }^{1} \cdot$ C. Azzopardi ${ }^{1}$ A. M. Davies ${ }^{1} \cdot$ S. L. James ${ }^{1} \cdot$ R. Botchu ${ }^{1}$
}

Received: 20 January 2020 / Accepted: 20 January 2020 / Published online: 5 February 2020

(C) Italian Society of Medical Radiology 2020

\begin{abstract}
There is wide variation in the technique and type of contrast used for MR arthrography of shoulder. In this article, we discuss the current practice in UK and the reason for the change over the last few years.
\end{abstract}

Keywords Arthrogram $\cdot$ Shoulder $\cdot$ UK

Direct MR arthrograms (MRA) utilise distension of the joint capsule to separate intra-capsular structures and hence improve visualisation, when compared to conventional non-arthrographic MRI. Clinical applications of direct MRA were first described in the shoulder, which remains one of the primary joints imaged with this technique. Although MRA of the shoulder is performed in many centres across the UK, there is variation in the way this study is performed, including whether gadolinium-based contrast agents (GdCAs), saline or other agents are used to distend the joint space.

An online survey was conducted of musculoskeletal radiologists in the UK to ascertain current practise in MRA of the shoulder, changes in practice in the last 5 years, and reasons for such changes. There have been recent concerns regarding gadolinium deposition in the brain and other tissues following enhanced MRI studies. In this commentary, we discuss the issues surrounding GdCA use in shoulder arthrograms, with reference to the literature and our survey findings.

\section{Widespread use of gadolinium-containing contrast agents in direct MRA of the shoulder}

Of the 78 respondents to the survey, all reported that they performed MRI arthrograms of the shoulder as part of their practice with $86 \%$ performing more than 20 studies each year. Totally, $94 \%$ of respondents used GdCAs in the injectate used to distend the glenohumeral joint space.

Historically, direct MR arthrography of the shoulder utilising either gadolinium and saline mixture, or saline alone was both initially described in the 1990s [1, 2]. A further technique known as indirect MR arthrography which relies on diffusion of GdCAs from synovium into the joint fluid following intravascular injection was also developed, but this does not provide the joint distension which is helpful for visualisation of intra-capsular structures [3]. Since then, direct MRA shoulder arthrography utilising GdCAs has become an established practice.

Medicines and Healthcare Regulatory Authority (MHRA) advice to health professionals in the UK states that GdCAs should be used 'only when diagnostic information is essential and not available with unenhanced magnetic resonance imaging' [4]. Given that distension of the shoulder joint for MRA can be achieved with normal saline and/or local anaesthetic, the justification for using GdCAs has to be considered.
R. Botchu

drbrajesh@yahoo.com

1 Department of Musculoskeletal Radiology, The Royal Orthopedic Hospital, Bristol Road South, Northfield, Birmingham, UK 


\section{Benefit of GdCA use in MRAs}

In 1997, Zanetti and Hodler [5] reported that shoulder MRAs using GdCAs were technically superior to saline arthrograms, using qualitative scoring system and when comparing signal-to-noise ratios. However, this study utilised different sequences between the MRAs with GdCAs and saline, and used imaging parameters that may not be comparable with current practice such as relatively low field strength (1.0T) and no fat suppression.

More recent studies have questioned the benefit of using GdCAs in MR arthrograms [6-8]. In contrast to Zanetti and Hodler [5], Singer et al. [6] compared MRAs with GdCAs against saline MRAs, and found no significant difference in diagnostic accuracy for labral and rotator cuff pathology with arthroscopy as the gold standard.

Even without GdCAs, there is intrinsic contrast between fluid injected into the glenohumeral joint during direct MR arthrograms and lower-signal intra-capsular structures on T2 and other fluid-sensitive sequences. The addition of GdCAs in the joint allows delineation of intra-capsular structures on T1-weighted sequences. The question therefore is whether these gadolinium T1-weighted sequences provide additional diagnostic information compared to fluid-sensitive sequences. Two studies investigating this question suggest that they do not. Helms et al. [7] reported comparable diagnostic accuracy for rotator cuff and labral pathology on MRAs of the shoulder using T1and T2-weighted sequences, and when using T2-weighted sequences alone. Similarly, Guermazi et al. [8] reported comparable diagnostic accuracy for labral and cartilage pathology on T1- and T2-weighted sequences.

\section{Safety concerns of gadolinium-containing contrast agent use}

Only two respondents cite concerns regarding GdCA safety as their reason for omitting GdCAs in MR shoulder arthrograms. This is not due to lack of awareness, as $77 \%$ were aware of the recent developments on gadolinium retention.

There is increasing evidence of the long-term deposition of gadolinium in the brain and other tissues following intravascular use of GdCAs, particularly linear agents $[9,10]$. Prolonged urinary excretion beyond $24 \mathrm{~h}$ occurs following administration, demonstrating slow release from a deep compartment within the body [11]. Excreted gadolinium compounds persist in the environment and have been detected in tap water, highlighting the pervasive use of GdCAs in imaging [12]. The precise nature of the gadolinium that is deposited within tissues (dechelated or bound gadolinium, or intact GdCA) has not been established, and therefore the toxicity and clinical consequences are unknown [13]. A research roadmap by NIH/ACR/RSNA workgroup was published in 2018 identifying important knowledge gaps about gadolinium chelates that need to be addressed to better understand the safety implications of gadolinium deposition [13]. The evidence for gadolinium deposition within the brain and body has resulted in withdrawal of licences of linear agents from intravascular use in the UK by the MHRA, which has been in effect since 1 February 2018 [4].

Importantly, the developing body of evidence for brain and other tissue gadolinium deposition relates to intravascular use, not when used intra-articularly as for MRAs. Brain deposition of gadolinium has not been demonstrated following intra-articular injection of GdCA [14]. Safety concerns regarding GdCAs are less of a concern in MRAs as the doses of GdCA used in direct shoulder MRAs are a tiny fraction of those used intravenously for contrast-enhanced studies. A GdCA dose of 20-50 nmol of would be typical for a MR shoulder arthrogram (10-20 $\mathrm{ml}$ of 2-2.5 mmol/L GdCA), representing approximately $1 / 200$ th the dose used intravenously in a body or brain MRI $(7 \mathrm{mmol}$ for a $70 \mathrm{~kg}$ patient at $0.1 \mathrm{mmol} / \mathrm{kg}$ ).

\section{Supply issues with GdCAs}

Ten radiologists in our survey reported changes in their practise with their use of GdCAs in MR arthrograms because of supply issues.

Gadopentetic acid, diglutamine salt (Magnevist) is the only GdCA which is licensed for intra-articular use in the UK. This is available as a glass pre-filled syringe containing $20 \mathrm{ml}$ of Magnevist at a concentration of $2 \mathrm{mmol} / \mathrm{l}$. However, there was a supply shortage of pre-filled Magnevist syringes in early 2019, prompting consideration of alternatives.

Magnevist was also previously available as an intravascular preparation at a concentration of $0.5 \mathrm{mmol} / \mathrm{ml}$ $(500 \mathrm{mmol} / \mathrm{L})$. This intravascular preparation could be diluted by a ratio of approximately 1:200 for intra-articular use. However, following the withdrawal of the UK licence for intravascular use of Magnevist, unexpired stocks of intravascular preparations of Magnevist were recalled [4]. Some survey participants reported diluting intravascular preparations of other (non-Magnevist) gadolinium compounds for use in MR arthrograms. This is technically feasible, but would be outside their UK licence of these gadolinium compounds, and thus be subject to local pharmacy policies on off-label use. Adherence to GMC guidance on unlicensed medications would be recommended in these instances [15]. 
There are currently no gadolinium-based agents that are licensed for intra-articular use in children in the UK.

\section{Impact of increased magnet field strength on MRI}

Two respondents cited the presence of 3 Tesla (3T) imaging as a reason to change their current practice with shoulder MRA. Improved image quality with $3 \mathrm{~T}$ imaging potentially allows better depiction of anatomy and pathology compared to $1.5 \mathrm{~T}$ imaging, although there are no studies directly comparing the diagnostic accuracy of shoulder MRAs performed at $1.5 \mathrm{~T}$ versus $3 \mathrm{~T}$.

Where 3T imaging is available, the additional diagnostic yield of MRAs over conventional MRIs may be less pronounced. Conventional MRI at 3T has comparable accuracy to MRAs at $3 \mathrm{~T}$ for rotator cuff tears, though MRAs have greater sensitivity for labral tears [16-18]. Magee [19] reported that the likelihood of finding an additional finding on MRA is small (6\%) if an initial non-arthrographic conventional MRI was abnormal.

\section{Varying experience with MR shoulder arthrograms performed with and without gadolinium}

At the authors' institution, all MR shoulder arthrograms have been performed without gadolinium in the past 2 years with satisfactory visualisation of the cartilage and labrum. In contrast, 4 survey respondents reported previously performing arthrograms without GdCAs due to supply issues, but have a preference for MR arthrograms with GdCAs due to concerns on imaging quality. The quality of the imaging achieved with and without the use of GdCAs may vary from centre to centre depending on the field strength, MR sequences and radiographer expertise.

\section{Conclusion}

The use of gadolinium-based agents for shoulder MRA is prevalent in the UK. However, a minority use saline and/ or local anaesthetic without GdCAs to achieve joint distension for this procedure in the UK. A discussion of the issues regarding GdCA use in MRA has been presented in this commentary. It is hoped that this commentary has shed light on the issues regarding GdCAs for MRA of shoulder, and provide a reference for radiologists who are considering their GdCAs use.
Funding No funding to declare.Ethical standards This article does not contain any studies with human participants or animals performed by any of the authors.

\section{Compliance with ethical standards}

Conflict of interest The authors declare that they have no conflict of interest.

\section{References}

1. Flannigan B, Kursunoglu-Brahme S, Snyder S, Karzel R, Del Pizzo W, Resnick D (1990) MR arthrography of the shoulder: comparison with conventional MR imaging. Am J Roentgenol 155(4):829-832. https://doi.org/10.2214/ajr.155.4.2119117

2. Tirman PFJ, Stauffer AE, Crues JV et al (1993) Saline magnetic resonance arthrography in the evaluation of glenohumeral instability. Arthroscopy 9(5):550-559. https://doi.org/10.1016/S0749 -8063(05)80403-7

3. Vahlensieck M, Lang P, Sommer T, Genant K, Schild H (1997) Indirect MR arthrography: techniques and applications. Semin Ultrasound CT MRI 18(4):302-306. https://doi.org/10.1016/ S0887-2171(97)80021-2

4. Gadolinium-containing contrast agents: removal of Omniscan and iv Magnevist, restrictions to the use of other linear agents. GOV.UK. https://www.gov.uk/drug-safety-update/gadolinium -containing-contrast-agents-removal-of-omniscan-and-iv-magne vist-restrictions-to-the-use-of-other-linear-agents. Accessed $8 \mathrm{Dec}$ 2019

5. Zanetti M, Hodler J (1997) Contrast media in MR arthrography of the glenohumeral joint: intra-articular gadopentetate vs saline: preliminary results. Eur Radiol 7(4):498-502. https://doi. org/10.1007/s003300050191

6. Singer AD, Rosenthal J, Umpierrez M, Guo Y, Gonzalez F, Wagner E (2019) A comparison of saline and gadolinium shoulder MR arthrography to arthroscopy. Skeletal Radiol. https://doi. org/10.1007/s00256-019-03338-2

7. Helms CA, McGonegle SJ, Vinson EN, Whiteside MB (2011) Magnetic resonance arthrography of the shoulder: accuracy of gadolinium versus saline for rotator cuff and labral pathology. Skeletal Radiol 40(2):197-203. https://doi.org/10.1007/s0025 6-010-0978-1

8. Guermazi A, Jomaah N, Hayashi D et al (2014) MR arthrography of the shoulder: optimizing pulse sequence protocols for the evaluation of cartilage and labrum. Eur J Radiol 83(8):1421-1428. https ://doi.org/10.1016/j.ejrad.2014.04.030

9. McDonald RJ, McDonald JS, Kallmes DF et al (2015) Intracranial Gadolinium deposition after contrast-enhanced MR imaging. Radiology 275(3):772-782. https://doi.org/10.1148/radiol.15150 025

10. Kanda T, Fukusato T, Matsuda M et al (2015) Gadolinium-based contrast agent accumulates in the brain even in subjects without severe renal dysfunction: evaluation of autopsy brain specimens with inductively coupled plasma mass spectroscopy. Radiology 276(1):228-232. https://doi.org/10.1148/radiol.2015142690

11. Lancelot E (2016) Revisiting the pharmacokinetic profiles of gadolinium-based contrast agents: differences in long-term 
biodistribution and excretion. Invest Radiol 51(11):691-700. https ://doi.org/10.1097/RLI.0000000000000280

12. Schmidt K, Bau M, Merschel G, Tepe N (2019) Anthropogenic gadolinium in tap water and in tap water-based beverages from fast-food franchises in six major cities in Germany. Sci Total Environ 687:1401-1408. https://doi.org/10.1016/j.scito tenv.2019.07.075

13. McDonald RJ, Levine D, Weinreb J et al (2018) Gadolinium retention: a research roadmap from the 2018 NIH/ACR/RSNA workshop on gadolinium chelates. Radiology 289(2):517-534. https:// doi.org/10.1148/radiol.2018181151

14. Kralik SF, Singhal KK, Frank MS, Ladd LM (2018) Evaluation of gadolinium deposition in the brain after MR arthrography. Am J Roentgenol 211(5):1063-1067. https://doi.org/10.2214/ AJR.18.19833

15. Prescribing unlicensed medicines. https://www.gmc-uk.org/ethic al-guidance/ethical-guidance-for-doctors/prescribing-and-manag ing-medicines-and-devices/prescribing-unlicensed-medicines. Accessed 9 Dec 2019

16. Ajuied A, McGarvey CP, Harb Z, Smith CC, Houghton RP (2018) Corbett SA (2018) Diagnosis of glenoid labral tears using 3-tesla MRI vs. 3-tesla MRA: a systematic review and metaanalysis. Arch Orthop Trauma Surg 138(5):699-709. https://doi. org/10.1007/s00402-018-2894-0

17. McGarvey C, Harb Z, Smith C, Houghton R, Corbett S, Ajuied A (2016) Diagnosis of rotator cuff tears using 3-Tesla MRI versus 3-Tesla MRA: a systematic review and meta-analysis. Skeletal Radiol 45(2):251-261. https://doi.org/10.1007/s0025 6-015-2299-x

18. Major NM, Browne J, Domzalski T, Cothran RL, Helms CA (2011) Evaluation of the glenoid labrum with 3-T MRI: is intraarticular contrast necessary? AJR Am J Roentgenol 196(5):11391144. https://doi.org/10.2214/AJR.08.1734

19. Magee T (2016) Utility of pre- and post-MR arthrogram imaging of the shoulder: effect on patient care. Br J Radiol 89(1062):20160028. https://doi.org/10.1259/bjr.20160028

Publisher's Note Springer Nature remains neutral with regard to jurisdictional claims in published maps and institutional affiliations. 